\title{
Cathodoluminescence hyperspectral analysis of whispering gallery modes in active semiconductor wedge resonators
}

\author{
Pierre Guillemé ${ }^{1,2}$, Julie Stervinou ${ }^{1}$, Tony Rohel ${ }^{1},{\text { Charles } \text { Cornet }^{1} \text {, David Gachet }}^{3}$,

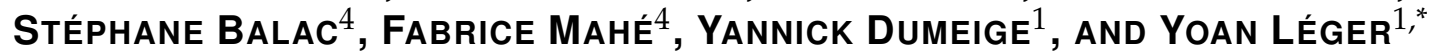 \\ ${ }^{1}$ Univ Rennes, INSA Rennes, CNRS, Institut FOTON - UMR 6082, F-35000 Rennes, France \\ ${ }^{2}$ Current address: Nanoscience Laboratory, Department of Physics, University of Trento, I-38123 Povo-Trento, Italy \\ ${ }^{3}$ Attolight AG, EPFL Innovation Park, Building D, 1015 Lausanne, Switzerland \\ ${ }^{4}$ IRMAR, CNRS UMR 6625, Université de Rennes 1, F-35042, Rennes, France \\ *Corresponding author: yoan.leger@insa-rennes.fr \\ Compiled April 19, 2018
}

Whispering gallery mode resonators are key devices for integrated photonics. Despite their generalization in fundamental and applied science, information on spatial confinement of light in these structures is mostly retrieved from purely spectral analysis. In this work, we present a detailed spectral and spatial characterization of whispering gallery modes in active semiconductor microdisk resonators by use of hyperspectral cathodoluminescence. By comparing our experimental findings to finite element simulations, we demonstrate that the combination of spectral and spatial measurements enables unique identification of the modes and even reveals specific features of the microresonator geometry such as a wedge profile.

Hyperspectral cathodoluminescence (HCL) consists in the recording and processing of 4D datasets containing the cathodoluminescence intensity spectrum of a sample for each pixel of the scanning electron microscopy image [1]. This method has now become the ultimate characterization tool in many research fields [2-5], demonstrating its potential through advanced spectral processing [3] and/or the combination of spectral and spatial representations [2]. More precisely, in the field of photonics, HCL has recently been used to measure the local density of optical states (LDOS) in photonic crystal cavities [2]. Nanometer scale information on the density of optical states in photonic devices is a key asset for the developement of future on-chip photonic devices [6], from laser sources [7] to nonlinear devices [8].

Nevertheless, such a technique has rarely been used for whispering gallery mode (WGM) resonators, despite their ubiquity in current photonic research, from opto-mechanics [9] and quantum optics [10] to bio-physics [11]. Most demonstrations of WGM cathodoluminescence images have been demonstrated with wide band gap materials such as $\mathrm{GaN}$ [12] and $\mathrm{ZnO}$ [13] while recourse to HCL remains anecdotal [5]. Conversely, recent results using scanning near-field microscopy on InP-based microdisks [14] demonstrate the vivid interest in imaging WGMs at the sub-wavelength resolution.

In this work, we fill this gap by reporting an advanced hyperspectral characterization of whispering gallery modes in an active gallium phosphide-based microdisk using low temperature cathodoluminescence. Our measurements experimentally reveal the nanoscale differences between mode geometries, allowing us to identify the radial order $n$ of the modes and even polarization states. Signature of the microdisk shape, namely a wedge rim, is also demonstrated unambiguously through the relative spatial distributions of $E_{z}$ and $H_{z}$ polarized modes.

Contrary to the work of Sapienza et al. [2] where the LDOS is measured within the band gap of a dielectric- 


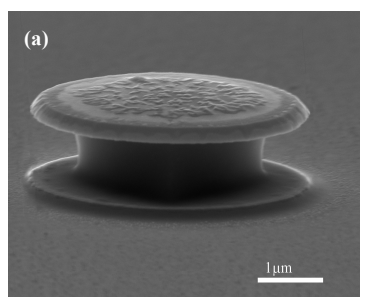

(c)

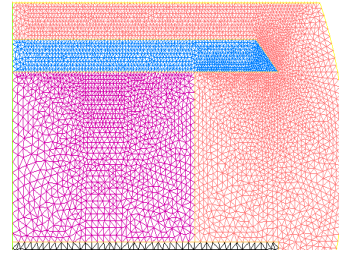

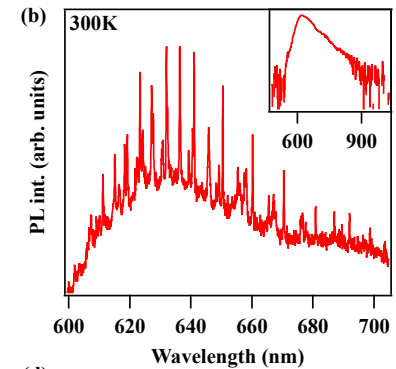

(d)

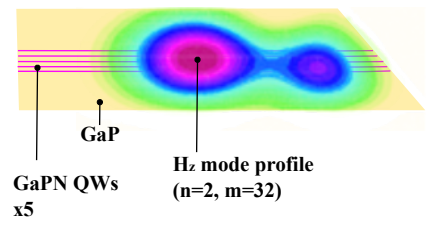

Fig. 1. a) Scanning electron microscopy image of an active $\mathrm{GaP}$ microdisk on $\mathrm{Al}_{0.7} \mathrm{GaP}$ pedestal. b) Room temperature photoluminescence of such disks; Emission from GaPN QWs is filtered by high Q $(\sim 10000)$ WGMs. The inset shows the logscale GaPN QW emission spectrum from the unprocessed sample, where a high wavelength exponential tail is clearly observed. c) Finite element mesh used to calculate the WGMs of the microdisks. d) Sketch presenting the overlap between the GaPN QWs and the $(n=2, m=32, p=1)$ WGM as extracted from the FEM calculation.

material photonic-crystal device, with the electron beam acting directly as a broadband dipole source, known as coherent excitation [15, 16], our measurements should more likely originate from incoherent excitation consisting in the creation and relaxation of many electron-hole pairs down to the active material of the photonic structure, namely quantum wells (QWs)and subsequent radiative recombination [17]. In some cases coherent emission processes can also be observed above the semiconductor band gap emission [18] and photon correlation experiments then become necessary to identify the coherent and incoherent contributions to the signal [19]. In the present work, the spectral range of the CL signal lying precisely at the position of the QW radiative transition as discussed in the following, we will consider that the dominant excitation process is mainly incoherent. Beside good emission properties, the recourse to such an excitation scheme to retrieve information on the LDOS requires to validate the following criteria: i) the homogeneity of the material optical properties; ii) the weak influence of carrier relaxation and diffusion to maintain a spatial resolution as close as possible to the electron beam profile; iii) the minimization of photon reabsorption in the photonic structure, due to the localized excitation scheme. Finally, the ideal material should present a large emission spectrum to probe as many WGMs as possible.

These criteria are mostly satisfied with dillute-nitride GaP-based materials. As far as emission homogeneity is concerned, semiconductor microdisks, and especially suspended geometries such as the one presented in Figure 1 a) are often affected by surface recombination losses. This problem is partially circumvented by the use of nanostructured materials (i.e. quantum wells in the present work) so that only the disk rims can alter the emission. In the case of GaP-based devices, the surface recombination rate is low, typically one order of magnitude smaller than in GaAs [20], limiting the degradation of the material emission properties in the vicinity of the rims. Because of its indirect band gap, GaP is intrinsically a poor light emitter. The incoporation of nitrogen in $\mathrm{GaP}$ in the dillute regime (a few \%) is associated with an important increase of the material emission properties because nitrogen states, strongly localizing electrons, get coupled with the GaP $\Gamma$ band to form a pseudo-direct band gap material [21]. The $\mathrm{GaPN}_{0.02}$ quantum wells (QWs) used in this work present room temperature photoluminescence originating from the band-to-band pseudo-direct transition between 600 and $900 \mathrm{~nm}$, well below the GaP band gap (550nm), as shown in the inset of figure $1 \mathrm{~b}$ ). At low temperature $(10 \mathrm{~K})$ where cathodoluminescence experiments are carried out, the Varshni band gap variation blueshifts the QW emission by $25 \mathrm{~nm}$ so that the PL/CL maximum lies around $615 \mathrm{~nm}$. A strong localization character is 
(a)

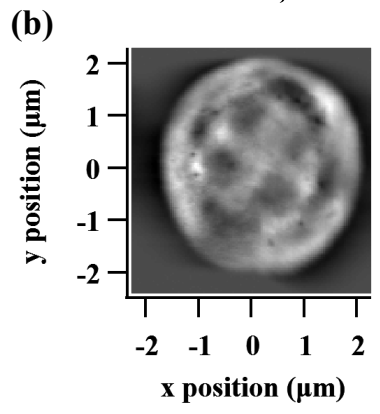

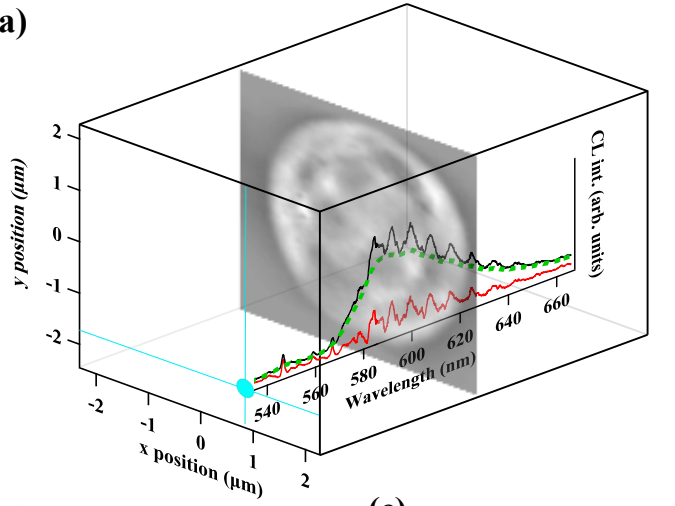

(c)

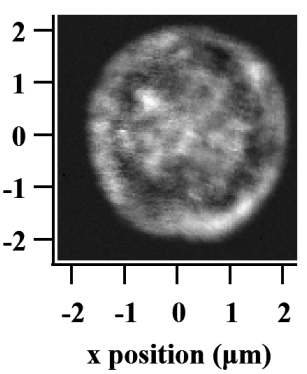

(d)

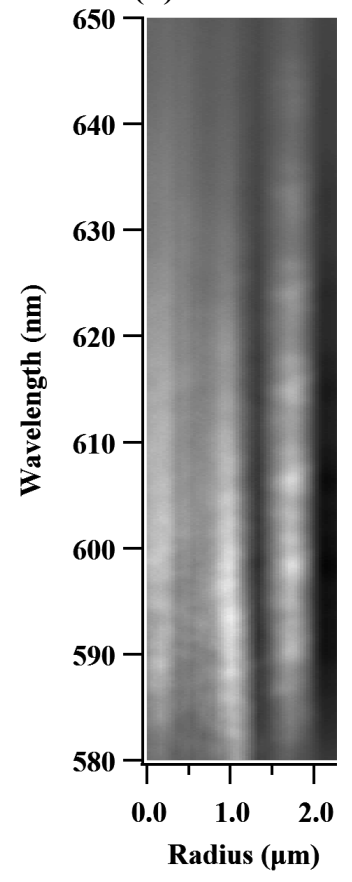

(e)

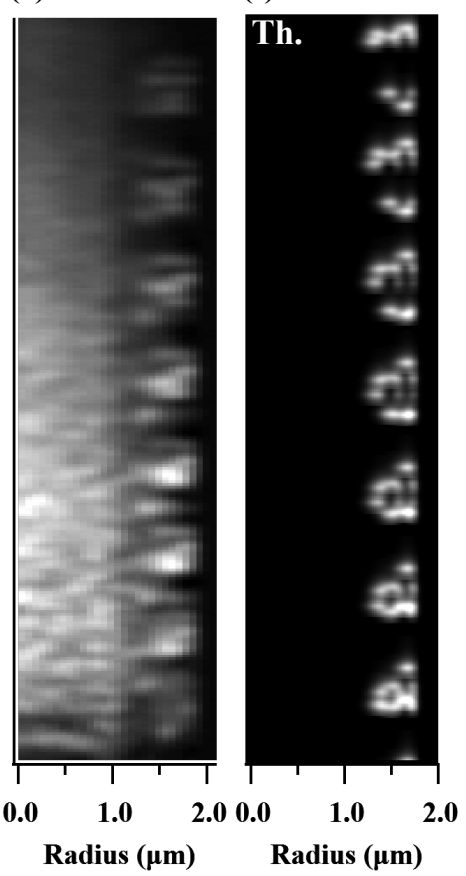

Fig. 2. a) Sketch of the hyperspectral data cube: HCL provides the emission intensity spectrum for each excitation point of an e-beam $x-y$ scan. The raw emission (black spectrum) can be processed to remove the background contribution (dashed green spectrum) from the WGMs contribution (red spectrum). Panels $b$ ) and c) show $x-y$ slices in the HCL cube at wavelength $\lambda=584.5 \mathrm{~nm}$ where a WGM is observed, before and after background filtering respectively. Panels $\mathrm{d}$ ) and e) are $\lambda-x$ slices of the HCL cube at $y=0$ before and after filtering as well. The data set presented throughout this article was obtained on a typical $4 \mu \mathrm{m}$ diameter microdisk. Panel f) shows the calculated $\lambda-x$ slice of the Poynting vector magnitude in a wedge microdisk, obtained by limiting the mode $\mathrm{Q}$ factor to a fixed value close to the experimental one.

conserved as evidenced by the high-wavelength exponential tail in the photoluminescence spectrum. This is a great advantage in the present work since: it limits the carrier diffusion length to $100 \mathrm{~nm}$ [22]; it protects the carriers from non-radiative relaxation channels and it is responsible for a large inhomogeneous broadening of the spectrum. Last but not least, localization leads to a non-negligeable Stokes shift [23] which limits reabsorption mechanisms. All these features reinforce the fact that the hyperspectral cathodoluminescence measurements reported in this work provide accurate information on the microdisk LDOS.

The GaP suspended microdisks presented here were fabricated from epilayers grown by molecular beam epitaxy. The heterostructure consists of a 200-nm-thick GaP layer containing five 3-nm GaPN 0.02 QWs deposited on top of a 1-micron-thick $\mathrm{Al}_{0.7} \mathrm{GaP}$ sacrificial layer, grown on a $\mathrm{GaP}$ substrate. E-beam lithography was used to define the microdisk structures using SU8 resist and a SiN ${ }_{x}$ hard mask. Chlorine-based dry etching and lateral wet etching with hydrofluoric acid were then used to obtain the suspended geometries, a SEM view of which is presented in figure $1 \mathrm{a}$ ). A wedge profile of the disk of $50^{\circ}$ is obtained due to the lateral etching of the hard mask during the dry etching process. Room-temperature microphotoluminescence on these microdisks reveals WGMs with $Q$ factors in the range of $10^{4}$ mainly limited by the rim roughness (10 nm RMS) as shown in figure $1 \mathrm{~b}$ ).

The HCL experiments were performed at low temperature $(10 \mathrm{~K})$ using an Attolight Allalin integrated cathodoluminescence microscope with a $100 \mathrm{~nm}$ spatial resolution and a spectral resolution of $0.1 \mathrm{~nm}$ on a 4 $\mu \mathrm{m}$-diameter microdisk. The beam voltage was set to $3 \mathrm{kV}$. Once the HCL data cube is recorded (see figure $2 \mathrm{a}$ ), a data processing routine is used to separate the background emission, dominated by low-Q Fabry-Perot modes (dashed green plot) from the WGM contribution (red spectrum). This routine is performed independently from one spatial pixel to the other but does not impact the spatial integrity of the data as shown in the $x-y$ 
hyperspectral slices presented in figure $2 \mathrm{~b}$ ) and c). These plots present the CL spatial distribution before and after filtering respectively on a WGM resonance at $\lambda=584.5 \mathrm{~nm}$. Even if noisier, the annular profile of the WGM at this wavelength is clearly conserved from b) to c) while the cross shape pattern at the center of the disk, induced by the square-base pedestal becomes less visible demonstrating the different origins of these emission structures. The effect of the background filtering is much more interesting along a $\lambda-x$ slice of the HCL cube, as demonstrated in figure $2 \mathrm{~d}$ ) and e). While the WGMs can only be barely observed before filtering (panel d), a structure is observed after filtering (panel e). Whispering gallery modes, lying along the rims of the microdisks $(r>1 \mu \mathrm{m})$, are clearly discriminated against emission in the central area, where high radial order WGMS and Fabry-Perot modes in the disk coexist. Different sets of WGMs can already be qualitatively identified through the analysis of their free spectral range (FSR) and radial extension. In the following, we quantitatively analyze these parameters to identify the different mode sets.

(a)

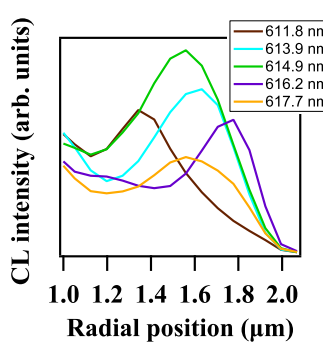

(c)

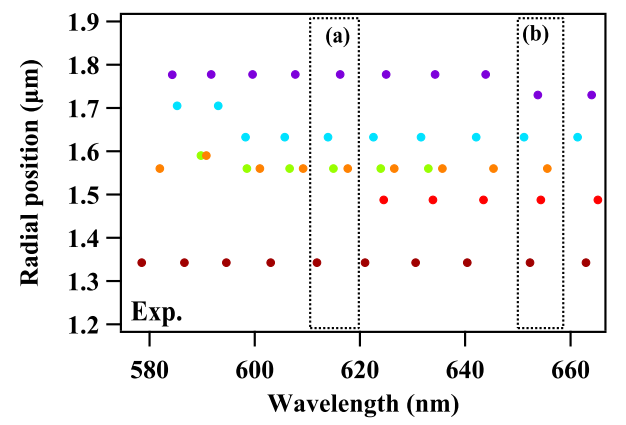

(b)

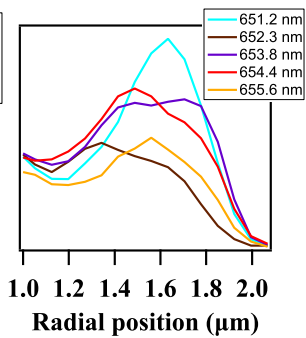

(e)

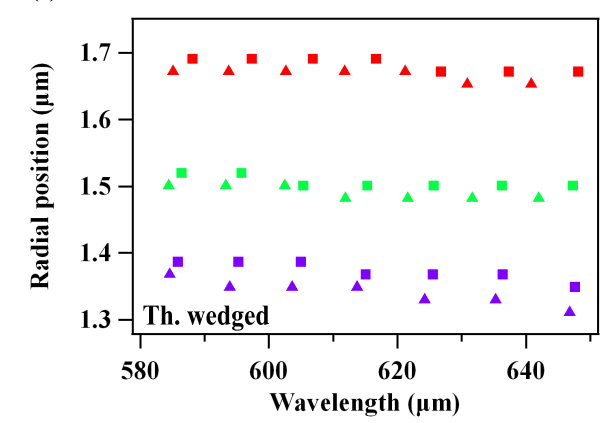

Fig. 3. a) and b) Spatial profiles of WGMs lying within two distinct wavelength ranges $(610-620 \mathrm{~nm}$ and $650-660 \mathrm{~nm}$ respectively). The color code for the modes is the same as the one used in graph c), presenting the spatial position of the mode maximum versus the resonance wavelength. The FSR for the different mode sets, from the external one to the internal one, is : $6.85 \mathrm{THz}$ (purple), $6.54 \mathrm{THz}$ (cyan),7.24 THz (orange), 6.95 $\mathrm{THz}$ (green), $7.36 \mathrm{THz}$ (red) and $7.34 \mathrm{THz}$ (brown). Panels d) and e) are the theoretical counterparts of c) obtained for a cylindrical disk and a wedge disk respectively. In the simulations, the disks' diameter is 4 $\mu \mathrm{m}$ in the two cases, with a wedge of $50^{\circ}$ for the second case. The FEM simulation allows to discriminate between $E_{z}$ and $H_{z}$ polarization as well as between the different radial orders $n$.

In figure 3 a) and b), we plot the spatial emission profiles of WGMs lying in two distinct wavelength ranges. In a) $(610-620 \mathrm{~nm}$ range), five WGMs are spectrally separated. Their spatial profiles show very distinct signatures but surprinsigly, they all present only one maximum, in opposition with the expectation for WGMs with different radial orders. One mode has an intensity maximum very close to the disk rim $(1.8 \mu \mathrm{m}$ for the $616.2 \mathrm{~nm}$ mode) while the $611.8 \mathrm{~nm}$ resonance features an intensity maximum closer to the disk center, at 1.35 $\mu \mathrm{m}$, with a long tail towards the microdisk rim. The three other resonances lie between these positions. We will see in the following that despite the absence of multiple lobes, these different modes can be attributed to different radial orders and polarizations. In some cases, two WGMs overlap, both spectrally and spatially. The combined information of the $\lambda-x$ slice is then of great advantage to differentiate the two modes. This is the case for the 653.8 and $654.4 \mathrm{~nm}$ modes presented in figure $3 \mathrm{~b}$ ), the maxima of which respectively lie at 1.48 
and $1.7 \mu \mathrm{m}$. For both intervals (a) and (b), the position of the maximum intensity for each mode is reported in figure $3 \mathrm{c}$ ) which was finally obtained by extending the previous spatial analysis to the whole emission spectrum. Six sets of WGMs can be identified from figure $3 \mathrm{c}$ ). Each set shows a distinct spatial position, except for the orange and green sets which both lie around $1.55 \mu \mathrm{m}$. The six sets also feature distinct FSRs (provided in the figure caption).

These experimental results are compared with eigenmode calculations obtained by the weak formulation of the finite element method (FEM) using Freefem++ [24]. A typical axi-symmetrical mesh, mimicking our $\mathrm{GaP}$ microdisks is presented in figure $1 \mathrm{c}$ ). From the eigenmode profiles, we extracted the magnitude of the Poynting vector at the height of the QWs, as shown in figure $1 \mathrm{~d}$ ). This value is expected to be proportional to the ideal cathodoluminescence signal, for fully localized carriers and in the absence of optical loss and non-radiative carrier recombination. Two disk geometries have been studied: a $50^{\circ}$ wedge-shaped microdisk, the calculated $\lambda-x$ slice of which is presented in figure $2 \mathrm{f}$ ) and a cylindrical disk. The WGM distributions for these two geometries are summarized in figures $3 \mathrm{~d}$ ) and e). As pointed out in figure 2, the experimental and calculated WGM distribution are at first sight qualitatively similar: WGMs with distinct spatial distributions allow to define six mode sets with FSR in the 10-nm range. In the simulations, WGMs show a number of lobes equal to their radial order, contrary to the experimental data. The secondary maxima for $n=2$ and $n=3$ radial order modes show roughly half the intensity of the main peak and are only $60-80 \mathrm{~nm}$ large. Their disappearance in the experiments in favor of a smooth signal decay (e.g. $611.8 \mathrm{~nm}$ mode in figure 3 a) may come from a larger carrier diffusion and also from carrier trapping by surface states at the disk rims.

In the calculations, the mode intensity maxima lie in the same spatial range as the experiment, between 1.3 and $1.8 \mu \mathrm{m}$ as shown in figures $3 \mathrm{~d}$ ) and e). If the six mode sets observed experimentally spread quite homogeneously within this span, the theoretical modes are grouped by polarization pairs around three distinct radial positions. The main difference between the two microdisk geometries precisely comes from the ordering of these pairs. For the cylindrical disk (figure $3 \mathrm{~d}$ ) ), the $E_{z}$ polarized modes are closer to the rims than the $H_{z}$ modes. The situation is reversed for the wedge microdisk (figure $3 \mathrm{e}$ )). The reader will notice that within a pair, the two polarization states present different FSRs: $E_{z}$ modes always feature a smaller one than $H_{z}$ modes. Back to the experimental results, we observe that the FSR alternance, from the more external modes to the more internal ones follows the one of a wedge disk (larger FSR, then smaller FSR and so on) in agreement with the SEM image of our structures. This, together with the spatial distribution of the mode sets allow us to characterize with good confidence the measured mode profiles: In figure $3 \mathrm{c}$ ), the purple and cyan mode sets are respectively the $H_{z}$ and $E_{z}$ modes with $n=1$ radial order; the green and orange sets are attributed to the $n=2 H_{z}$ and $E_{z}$ mode sets while the red and brown sets are attributed to the $n=3 H_{z}$ and $E_{z}$ modes respectively. The small deviations between the experimental mode distribution (figure $3 \mathrm{c}$ ) and the theoretical one (figure 3 e) can be explained by the interplay between the wedge and pedestal influences, extremely dependent on geometry variations.

As a conclusion, we demonstrated that hyperspectral cathodoluminescence in active photonic devices such as microdisks can provide the blueprint of the confined optical modes. This method enables the identification of WGM features such as radial order or polarization state. It also enlightens effects of geometry refinements such as a wedge profile on the mode distribution. Our results show the pertinence of hyperspectral cathodoluminescence as an ultimate characterization tool for advanced photonic engineering.

\section{REFERENCES}

1. J. Christen, J. Vac. Sci. \& Technol. B: Microelectron. Nanometer Struct. 9, 2358 (1991).

2. R. Sapienza, T. Coenen, J. Renger, M. Kuttge, N. F. van Hulst, and A. Polman, Nat Mater 11, 781 (2012).

3. C. M. MacRae, N. C. Wilson, and A. Torpy, Min. Petrol 107, 429 (2013).

4. P. R. Edwards, D. Sleith, A. W. Wark, and R. W. Martin, J. Phys. Chem. C 115, 14031 (2011).

5. J. Bruckbauer, P. R. Edwards, T. Wang, and R. W. Martin, Appl. Phys. Lett. 98, 141908 (2011).

6. A. W. Schell, P. Engel, J. F. M. Werra, C. Wolff, K. Busch, and O. Benson, Nano Lett. 14, 2623 (2014).

7. G. Crosnier, D. Sanchez, S. Bouchoule, P. Monnier, G. Beaudoin, I. Sagnes, R. Raj, and F. Raineri, Nat. Photonics 11, 297 (2017).

8. D. P. Lake, M. Mitchell, H. Jayakumar, L. F. dos Santos, D. Curic, and P. E. Barclay, Appl. Phys. Lett. 108, 031109 (2016).

9. C. Baker, W. Hease, D.-T. Nguyen, A. Andronico, S. Ducci, G. Leo, and I. Favero, Opt. Express 22, 14072 (2014).

10. E. Stock, F. Albert, C. Hopfmann, M. Lermer, C. Schneider, S. Höfling, A. Forchel, M. Kamp, and S. Reitzenstein, Adv. Mater. 25, 707 (2013).

11. D. Gandolfi, F. Ramiro-Manzano, F. J. Aparicio Rebollo, M. Ghulinyan, G. Pucker, and L. Pavesi, Sensors. 15, 4796 (2015). 
12. C. Tessarek, M. Heilmann, and S. Christiansen, physica status solidi (c) 11, 794 (2014).

13. S. Choi, C. Ton-That, M. R. Phillips, and I. Aharonovich, Appl. Phys. Lett. 103, 171102 (2013).

14. A. V. Shelaev, A. M. Mintairov, P. S. Dorozhkin, and V. A. Bykov, J. Physics: Conf. Ser. 741, 012132 (2016).

15. M. Kociak and L. F. Zagonel, Ultramicroscopy. 176, 112 (2017).

16. F. J. García de Abajo and L. A. Blanco, Phys. Rev. B 67, 125108 (2003).

17. S. Meuret, T. Coenen, H. Zeijlemaker, M. Latzel, S. Christiansen, S. Conesa-Boj, and A. Polman, Phys. Rev. B 96, 035308 (2017).

18. L. H. G. Tizei, L. F. Zagonel, M. Tencé, O. Stéphan, M. Kociak, T. Chiaramonte, D. Ugarte, and M. A. Cotta, J. Physics: Condens. Matter 25, 505303 (2013).

19. S. Meuret, L. Tizei, T. Cazimajou, R. Bourrellier, H. Chang, F. Treussart, and M. Kociak, Phys. Rev. Lett. 114, 197401 (2015).

20. A. G. Baca and C. I. H. Ashby, Fabrication of GaAs devices, no. 6 in Processing series (Inst. of Electrical Engineers, 2005).

21. R. Kudrawiec, A. Luce, M. Gladysiewicz, M. Ting, Y. Kuang, C. Tu, O. Dubon, K. Yu, and W. Walukiewicz, Phys. Rev. Appl. 1, 034007 (2014).

22. S. R. Kurtz, A. A. Allerman, C. H. Seager, R. M. Sieg, and E. D. Jones, Appl. Phys. Lett. 77, 400 (2000).

23. S. Ilahi, S. Almosni, F. Chouchane, M. Perrin, K. Zelazna, N. Yacoubi, R. Kudrawiec, P. Râle, L. Lombez, J.-F. Guillemoles, O. Durand, and C. Cornet, Sol. Energy Mater. Sol. Cells 141, 291 (2015).

24. F. Hecht, J. Numer. Math. 20, 251 (2013). 